\title{
Impact of Wind Penetration and HVDC Upgrades on Dynamic Performance of Future Grids
}

\author{
Nilanjan Ray Chaudhuri, Member, IEEE, Balarko Chaudhuri, Member, IEEE
}

\begin{abstract}
Impact of DFIG based wind penetration on the dynamic performance of future grids is studied in this paper. Both $A C$ and a mix of AC and HVDC transmission are considered. In general, DFIGs are found to contribute positively to system damping although the effect could be opposite depending on the operating condition. For deeper understanding, various effects contributing to the overall system damping (e.g. wind penetration at sending vs receiving end etc.) are studied in isolation keeping the other factors (e.g. tie-flow etc.) constant. Implications of supplying bulk of the future load growth by wind energy with only $\mathrm{AC}$ and both $\mathrm{AC}$ and HVDC are analyzed and compared. Observed trends are explained in terms of damping torque contribution of synchronous generators which is the primary source of damping in power systems. Power oscillation damping (POD) control action through supplementary modulation of DFIG rotor currents (both direct and quadrature axes) or active and reactive power order of VSC HVDC link is demonstrated.
\end{abstract}

Index Terms-Wind, Doubly-fed induction generator (DFIG), High voltage direct current (HVDC), Voltage source converter (VSC), Dynamic performance, Damping, Frequency, Synchronous Generator (SG), Power oscillation damping (POD),

\section{List OF ABBREVIATIONS AND NOTATIONS}

$\begin{array}{ll}\text { HVDC } & \text { High voltage direct current } \\ \text { VSC } & \text { Voltage source converter } \\ \text { SG } & \text { Synchronous generator } \\ \text { DFIG } & \text { Doubly-fed induction generator } \\ \text { POD } & \text { Power oscillation damping } \\ \text { MPPT } & \text { Maximum power point tracking } \\ \text { SISO } & \text { Single input single output } \\ \text { SIMO } & \text { Single input multiple output } \\ & \text { superscript for parameters } \\ & \text { in modified reference frame } \\ * & \text { superscript for reference parameters } \\ \omega_{r} / \omega_{t} & \text { DFIG/turbine rotor angular speed } \\ \omega_{s} & \text { synchronous speed } \\ \omega_{e l} & \text { base electrical speed, 377 rad/s } \\ \theta_{t w} & \text { angle of twist in turbine shaft } \\ k_{s h} & \text { shaft stiffness } \\ c_{s h} & \text { damping coefficient } \\ H_{g} / H_{t} & \text { DFIG/turbine inertia } \\ T_{e} / T_{m} & \text { DFIG electrical/turbine mechanical torque } \\ \psi_{s} & \text { DFIG stator flux } \\ \psi_{q s}^{\prime} & q \text {-axis DFIG stator flux }\end{array}$

Support from the EPSRC UK under grant EESC P11121 is gratefully acknowledged.

N. R. Chaudhuri and B. Chaudhuri are with the Control and Power Research group, Imperial College London, London, UK (e-mail: n.chaudhuri@imperial.ac.uk,b.chaudhuri@imperial.ac.uk).

$\begin{array}{ll}L_{s s}, L_{r r}, L_{m} & \text { DFIG stator, rotor and mutual inductance } \\ i_{d r}^{\prime} / i_{q r}^{\prime} & d / q \text {-axis DFIG rotor current } \\ i_{d s}^{\prime} / i_{q s}^{\prime} & d / q \text {-axis DFIG stator current } \\ i_{m s} & \text { DFIG magnetizing current } \\ v_{d r}^{\prime} / v_{q r}^{\prime} & d / q \text {-axis rotor voltage of DFIG } \\ R_{s} / R_{r} & \text { DFIG stator/rotor resistance } \\ s_{l} & \text { rotor slip } \\ K_{o p t} & \text { co-efficient of maximum power extraction } \\ v_{d t}^{\prime} / v_{q t}^{\prime} & d / q \text { - axis HVDC converter terminal voltage } \\ i_{d}^{\prime} / i_{q}^{\prime} & d / q \text { - axis HVDC converter current } \\ R_{c}, L_{c} & \text { HVDC converter transformer } \\ & \text { resistance and inductance } \\ E_{a c} & \text { voltage at the network side } \\ m_{d}^{\prime} / m_{q}^{\prime} & \text { of converter transformer } \\ V_{d c}, C & d / q \text { - axis modulation indices } \\ R_{L}, L_{L}, I_{d c} & \text { dc bus voltage and capacitance } \\ P_{r e c} / P_{i n v} & \text { rectifier/inverter end active power } \\ Q_{r e c} / Q_{i n v} & \text { rectifier/inverter end reactive power }\end{array}$

\section{INTRODUCTION}

$\mathbf{F}$ UTURE grids are envisaged to be made up of significant amounts of wind generation with a mix of $\mathrm{AC}$ and HVDC transmission. With increasing penetration of both asynchronous generation (primarily wind) and transmission (HVDC), it is imperative to understand the implications on system stability and dynamic performance. Of particular, although not only, interest is the impact on the damping and frequency of low frequency electro-mechanical oscillations.

Several papers have looked into effect of wind penetration on small-signal stability of AC systems [1], [2], [3], [4]. In [1], [5] typical operational situation was considered with a fixed system load being supplied with different relative share of SG and DFIG representing variations in wind speeds. Damping contribution of DFIG when compared to SG was found to be higher or lower depending on the operating condition in [1]. However, in [5], DFIG was always found to contribute positively to system damping. Beside the different nature of the two test cases in [1], [5] the change in tie-line flow was perhaps also a contributing factor. To ascertain, case studies with fixed load and constant tie-flow but different levels of wind penetration at sending and receiving ends are studied here. In [2], a futuristic scenario where increasing load would be supplied by DFIGs keeping the SG outputs constant was considered. In one of the case studies, the increasing loads were radially supplied by the DFIGs with little influence on the 
rest of the system. Here a more realistic situation is considered with the DFIG supplying increasing loads not only in its own area but another remote area resulting in varying tie-line transfer.

Transmission of large amounts of wind power would require new transmission facilities and/or reinforcement of the existing ones. Besides conventional AC technology, significant proportion of the transmission upgrade would be HVDC either to capture deep-sea offshore wind power or interconnecting different geographical regions to tackle wind intermittency problem. Presence of HVDC links is likely to influence the system dynamic behavior and at the same time present significant control opportunities. In this respect, both CSC as well as VSC [6], [7] options have been reported in the literature but mostly in the context of conventional (SG based) generation.

Future power systems are likely to have large amount of wind generation with a mix of $\mathrm{AC}$ and HVDC transmission. Therefore, the impact of a mix of synchronous and asynchronous generation and transmission on the dynamic performance of the system needs to be investigated together in an unified framework. The dynamic interaction among the above components is complex and is influenced by a variety of factors. In this paper, a simple test system is considered with a view to isolate the effects, identify and explain the trends under different scenarios. Also power oscillation damping control with supplementary modulation of DFIG rotor currents (both direct and quadrature axes) or active and reactive power order of VSC HVDC is demonstrated.

The critical contributions of this paper are:

1) Study the impact of supplying bulk of the future load growth through wind (DFIG) as opposed to conventional (SG) generation and develop deeper understanding by considering various effects contributing to the overall system damping (e.g. wind penetration at sending vs receiving end etc.) in isolation keeping the other factors (e.g. tie-flow etc.) constant.

2) Analyze the effect of mixed AC/DC transmission system on dynamic performance with SG/DFIG.

3) Explain the trends in dynamic performance in terms of damping torque contributions from different generators where the damping torque estimation is done with a novel phasor technique [8].

4) Illustrate power oscillation damping using modulation of both the $\mathrm{d}$ and q-axes components of the rotor current with a single input multi output (SIMO) state-feedback controller unlike the single input single output (SISO) controllers reported in literature. A remote feedback signal is used due to higher observability of the interarea mode.

\section{MODELING}

\section{A. DFIG}

The overall structure of a DFIG is shown in Fig. 1 (a) where an aggregated model of the wind farm was adopted [1]. Modelling of DFIG was done in synchronously rotating $d-q$ reference frame [9] with $d$-axis leading the $q$-axis as per IEEE convention, see Fig. 1 (b). The stator transients of the machine were neglected, the converters were assumed to be ideal and the dc link dynamics was also neglected as suggested in [5] - further details can be found in [10]. Besides the standard differential and algebraic equations used to model the generator [10], a two-mass model of the turbine and drive train was considered to take the torsional mode into account. The equations are not repeated here due to space restriction.

1) Rotor Side Converter (RSC) Control: Standard vector control approach [11] was adopted where the $q$-axis was aligned with $\psi_{s}$, see Fig. 1 (b). All notations in the modified reference frame are henceforth denoted with a prime. Therefore,

$$
\begin{aligned}
& L_{s s} i_{d s}^{\prime}+L_{m} i_{d r}^{\prime}=0 \Rightarrow i_{d s}^{\prime}=-\frac{L_{m}}{L_{s s}} i_{d r}^{\prime} \\
& \psi_{q s}^{\prime}=L_{s s} i_{q s}^{\prime}+L_{m} i_{q r}^{\prime}
\end{aligned}
$$

Neglecting $R_{s}$ and assuming $i_{m s}$ constant we can write,

$$
\psi_{q s}^{\prime} \approx L_{m} i_{m s} \Rightarrow i_{q s}^{\prime}=\frac{L_{m}}{L_{s s}}\left(i_{m s}-i_{q r}^{\prime}\right)
$$

This results in simplification of $v_{d r}^{\prime}$ and $v_{q r}^{\prime}$ as follows:

$$
\begin{gathered}
v_{d r}^{\prime}=-R_{r} i_{d r}^{\prime}-\sigma L_{r r} \frac{d\left(i_{d r}^{\prime}\right)}{d t}-s_{l} \omega_{s} \sigma L_{r r} i_{q r}^{\prime}-s_{l} \omega_{s} \frac{L_{m}^{2}}{L_{s s}} i_{m s} \\
v_{q r}^{\prime}=-R_{r} i_{q r}^{\prime}-\sigma L_{r r} \frac{d\left(i_{q r}^{\prime}\right)}{d t}+s_{l} \omega_{s} \sigma L_{r r} i_{d r}^{\prime}
\end{gathered}
$$

where, $\sigma=\left(1-\frac{L_{m}^{2}}{L_{s s} L_{r r}}\right)$. As shown in Fig. 1 (c) 'PLANT', the above equations can be rewritten in terms of $v_{d r}^{\prime \prime}$ and $v_{q r}^{\prime \prime}$ after isolating the disturbance terms: $s_{l} \omega_{s} \sigma L_{r r} i_{d r}^{\prime}$ and $s_{l} \omega_{s}\left(\sigma L_{r r} i_{q r}^{\prime}+\frac{L_{m}}{L_{s s}} i_{m s}\right)$, respectively. Values of the parameters used for modelling are shown in Table Iin the Appendix.

Note that $i_{d r}^{\prime}$ and $i_{q r}^{\prime}$ are measurable parameters whereas $i_{m s}$ can be estimated from (3). Therefore the measurable disturbances were used as feed-forward terms with appropriate signs to achieve decoupling between $d$ and $q$ axes current control loops. The torque reference was generated through MPPT which in turn determined $i_{d r}^{\prime *}$ as shown below:

$$
T_{e}^{*}=\left(\frac{L_{m}^{2}}{L_{s s}}\right) i_{m s} i_{d r}^{*}=K_{o p t} \omega_{r}^{2}
$$

$i_{q r}^{\prime *}$ was decided based on terminal voltage control with a droop of $K_{v c}$ while supplying the magnetizing current from RSC.

Note that moderate closed loop bandwidth (BW) is adequate in tracking $i_{d r}^{\prime *}$ and $i_{q r}^{\prime *}$ since they are dc in nature under steady state. Thus a BW of $300 \mathrm{rad} / \mathrm{s}$ was considered while designing the controller $K(s)$ as shown in the Table II in the Appendix.

2) Grid Side Converter (GSC) Control: The GSC was assumed to be lossless, i.e. the same real power flows through RSC and GSC. On the other hand $Q_{G S C}$ was kept zero to attain minimum converter size.

\section{B. VSC HVDC}

1) Converter Modelling: Sinusoidal PWM was considered for VSC HVDC system and the converters were represented by their averaged model. The converter model is shown in Fig. 2 (a) in a synchronously rotating reference frame $d^{\prime}-q^{\prime}$ where its 

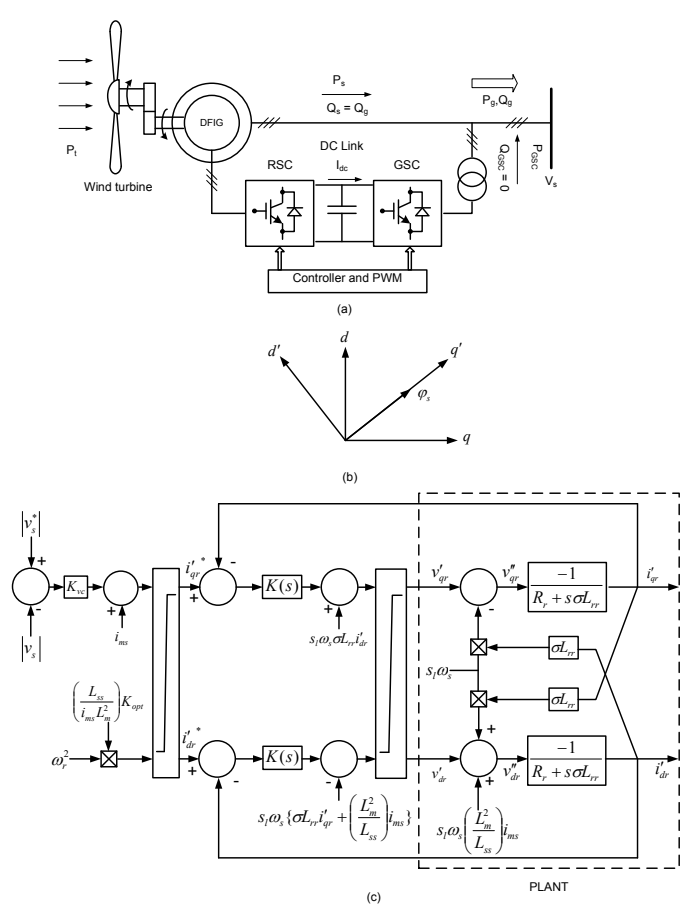

Fig. 1. (a) DFIG overall structure (b) $d-q$ : reference frame used for power system modelling, $d^{\prime}-q^{\prime}$ : modified reference frame for vector control (c) rotor converter control

$d$-axis coincides with $E_{a c}$, see Fig. 2 (b). Considering current injection model at both converter ends we can write:

$$
\begin{gathered}
v_{d t}^{\prime}-R_{c} i_{d}^{\prime}-L_{c} \frac{d i_{d}^{\prime}}{d t}+L_{c} \omega_{e l} i_{q}^{\prime}=E_{a c} \\
v_{q t}^{\prime}-R_{c} i_{q}^{\prime}-L_{c} \frac{d i_{q}^{\prime}}{d t}-L_{c} \omega_{e l} i_{d}^{\prime}=0
\end{gathered}
$$

where, $v_{d t}^{\prime}=m_{d}^{\prime} \frac{V_{d c}}{2}$ and $v_{q t}^{\prime}=m_{q}^{\prime} \frac{V_{d c}}{2}$. The nominal turns ratio of the converter transformer was designed to be 0.9 .

2) DC Link Modelling: The dc link was modelled by a lumped resistance and inductance:

$$
V_{d c r e c}-V_{d c i n v}=R_{L} I_{d c}+L_{L} \frac{d I_{d c}}{d t}
$$

DC bus dynamics at each converter end was modelled with differential equation showing power exchange in the capacitors. Fig. 2 (d) shows the inverter dc bus model as written below:

$$
P_{i n v}^{\prime}-P_{i n v}-i_{i n v}^{\prime 2} R_{c}=\frac{1}{2} C \frac{d\left(V_{d c i n v}^{2}\right)}{d t}
$$

where, $P_{i n v}^{\prime}=V_{d c i n v} I_{d c}, P_{i n v}=E_{a c i n v} i_{d i n v}^{\prime}$ and $i_{i n v}^{\prime 2}=$ $i_{\text {dinv }}^{\prime 2}+i_{\text {qinv }}^{\prime 2}$.

All HVDC parameters are shown in Table III in the Appendix.

3) Rectifier Control: The rectifier operates in $P-Q$ mode with decoupled control strategy[12] in the modified reference frame, see Fig. 2 (a)-(c). In line with DFIG current control, see Section II-A1 the controllers $K_{I}(s)$ were designed for a closed loop BW of $300 \mathrm{rad} / \mathrm{s}$.
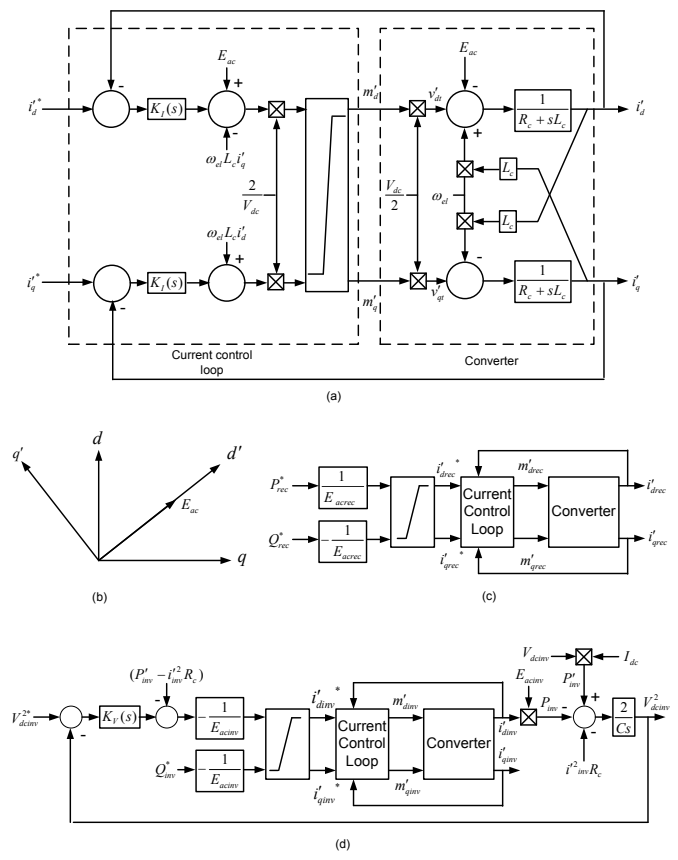

Fig. 2. (a) VSC HVDC converter model with overall control structure (b) modified reference frame for decoupled control (c) rectifier control (d) inverter control

4) Inverter Control: The inverter operates in $V_{d c}-Q$ mode maintaining constant dc bus voltage and unity power factor on the point of common coupling (PCC), see Fig. 2 (d). The outer voltage control loop is slower than the inner current control loop and consists of the feed-forward terms to mitigate measurable disturbances. A lead compensator $K_{V}(s)=0.027\left(\frac{s+231}{s+398.6}\right)$ providing a phase margin of 60 degrees at about $300 \mathrm{rad} / \mathrm{s}$ was employed to control the $\mathrm{dc}$ link voltage.

\section{TEST SYSTEM}

The well-known 4-machine, 2-area system [9] was considered for the case study. There are four generators, two (G1, G2 and G3, G4) in each area as shown in Fig. 3 (a). Generator G2 and G4 was considered to be either a SG or a DFIG. The SGs were represented by sub-transient models and were equipped with IEEE DC1A excitation systems. The DFIG was modeled as described earlier in Section II-A. The active component of the loads at buses 7 and 9 have constant current characteristics while the reactive component have constant impedance characteristics. The powerflow and dynamic data for the system can be found in [9].

As shown in Fig. 3 (b), a point-to-point VSC HVDC link was added in place of one of the tie-lines in the corridor between buses 7 and 9. Modeling and associated details of the VSC HVDC system is given in Section II-B.

\section{Case Studies}

There is one poorly damped inter-area mode and two local modes. To investigate the impact of wind (DFIG) penetration 

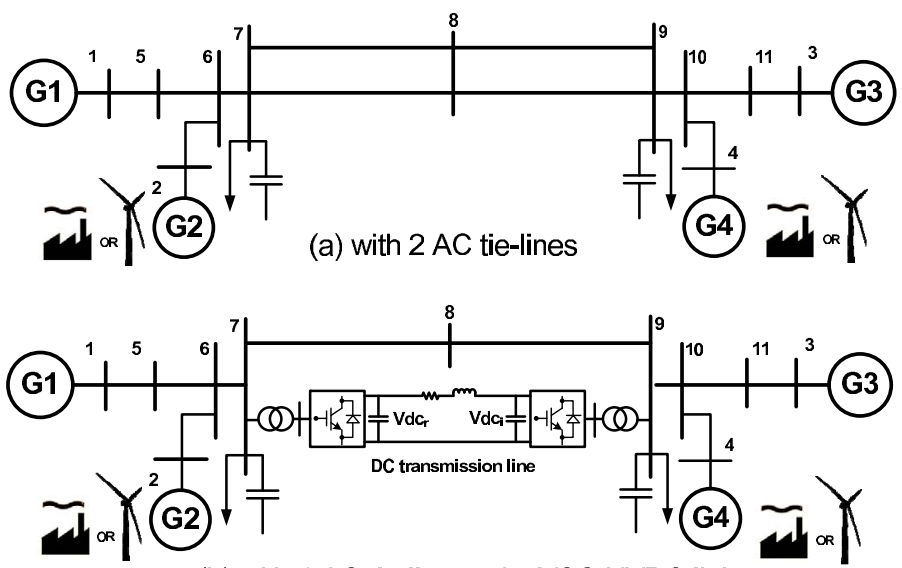

(b) with $1 \mathrm{AC}$ tie-line and a VSC HVDC link

Fig. 3. 4-machine, 2-area system with a) $2 \mathrm{AC}$ tie-lines and b) $1 \mathrm{AC}$ tie-line and a VSC HVDC link. Generator G2 and G4 could be either SG or DFIG

and VSC HVDC upgrade on the the inter-area mode, three broad scenarios as mentioned below were considered:

1) Relative proportion of DFIG and SG output power was changed to supply a fixed load (about $2800 \mathrm{MW}$ ) keeping the tie-line flow (400 MW) constant. Also DFIG at both sending end (SE), G2 and receiving end (RE), G4 were considered. This mimics a typical operational situation where a given system load is shared between the SGs and DFIGs in certain proportion according the available wind energy (speed).

2) Increase in system load (at both ends) was supplied by the DFIG, G2 while maintaining the outputs of SGs, G1, G3 and G4 almost unchanged. This is representative of a future planning scenario where bulk of the load growth is to be catered by wind energy.

3) AC corridor between area 1 and area 2 was upgraded by replacing one of the AC tie-lines with a VSC HVDC link.

Impact of DFIG on the inter-area mode was analyzed by comparing above situations against those with equivalent SG. Also the system behavior with both AC and VSC HVDC was compared against the case with only AC tie-lines. The above scenarios are discussed one by one in the next three subsections.

\section{A. Fixed Load and tie-flow, different mix of SG and DFIG}

In order to investigate the impact of DFIGs under a typical operational scenario, different mix of SGs and DFIG were considered keeping the system load and tie-flow constant. For each operating condition all generators were first modeled as SGs and then depending on the wind flow pattern either G2 (for SE) or G4 (for RE) or both were replaced by DFIG. Each operating condition corresponds to different levels of percentage contribution from DFIG which is compared against the same power being delivered by SG at those locations. The comparison is based on damping and frequency of the interarea mode which will be termed as 'system damping ratio' and 'system frequency' henceforth.

Figs 4 and 5 show the system damping ratio and system frequency for five scenarios with different levels of wind penetration from either end of the network expressed as a percentage of the overall system load. The general trend shows that DFIG increases the system damping and frequency when compared to equivalent synchronous generation.

Damping contribution of DFIG increases when it is located at RE rather than SE for the same DFIG output. The reason behind this is analyzed in terms of damping torque contribution from each of the SGs as elaborated in the later half of this Section. The increase in frequency with wind penetration is due to reduction in equivalent generator inertia as a result of 'decoupling' of DFIG rotor dynamics from the rest of the system. Note that the system frequency is the highest when $50 \%$ wind penetration is considered (25\% at each end) where G2 and G4 hardly contribute to inertia.

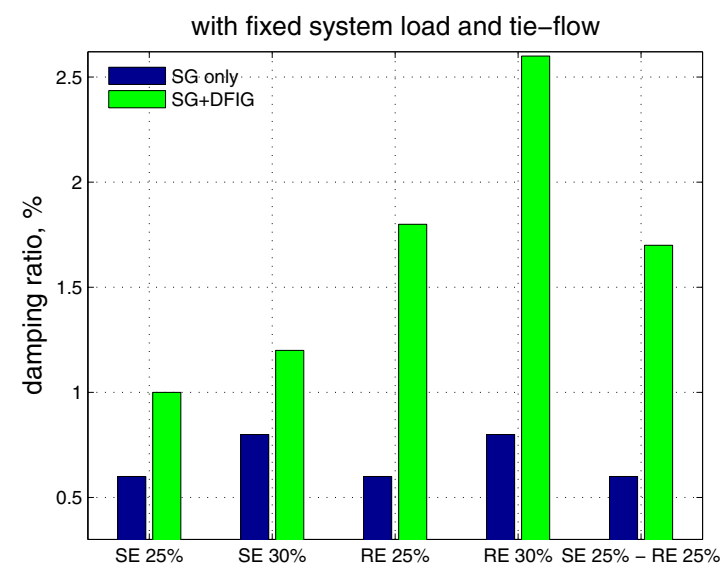

Fig. 4. Damping ratios of inter-area mode under different scenarios with fixed system load (2800 MW) and tie-flow (400 MW). Wind penetration (in $\%$ of system load) considered at sending end (SE) at G2 and receiving end (RE) at G4

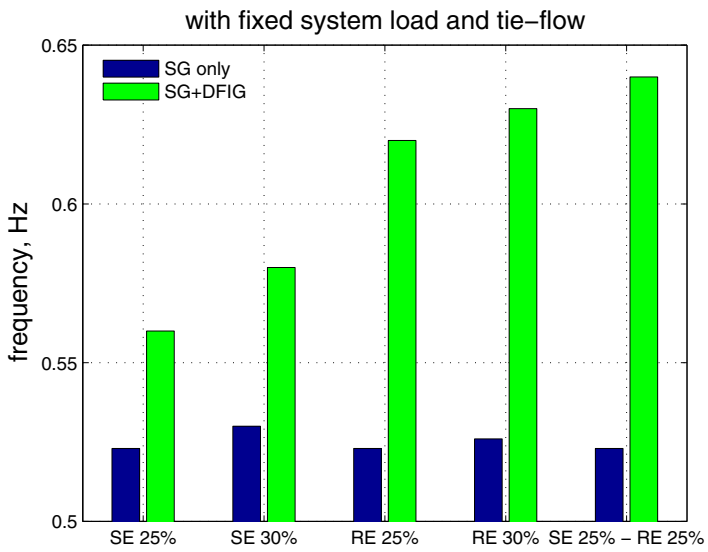

Fig. 5. Frequency of inter-area mode under different scenarios with fixed system load (2800 MW) and tie-flow (400 MW). Wind penetration (in \% of system load) considered at sending end (SE) at G2 and receiving end (RE) at $\mathrm{G} 4$

To gain insight into the increased system damping in presence of DFIG and their relative magnitudes, damping torque contributions from each generator were computed from simulation results. The oscillations in $T_{e}$ and $\omega$, as a result of a small disturbance in the system, were expressed as phasors and 
their relative magnitudes and angles were computed using the approach reported in [8]. Note that the torque phasor component of a generator along the direction of the correspondiong speed phasor when normalized with respect to the latter gives damping torque.

It can be seen from Fig. 6 that the net damping torque contribution from the SGs in each scenario is higher when DFIG is present. It was found that the DFIG rotor speed and torque variation were negligible compared to those of SGs which is also reflected in almost zero participation of DFIG in the inter-area mode - thereby contributing no damping torque on their own. For example when G2 and G4 are DFIGs the corresponding damping torque contributions are zero, see bottom right subplot of Fig. 6. However, the damping contribution of G1 and G3 increases significantly compared to the case where all are SGs - thereby producing larger damping ratio. Similar trend is visible for other scenarios. Moreover, the relative magnitudes of damping ratios for different scenarios is consistent with the trend of net $T_{d}$ values - e.g. $30 \%$ wind penetration at RE shows highest damping ratio $(\sim 2.5 \%)$ due to the largest net $T_{d}$ of $\sim 0.1 \mathrm{pu}$-s/rad compare Figs 4 and 6 .
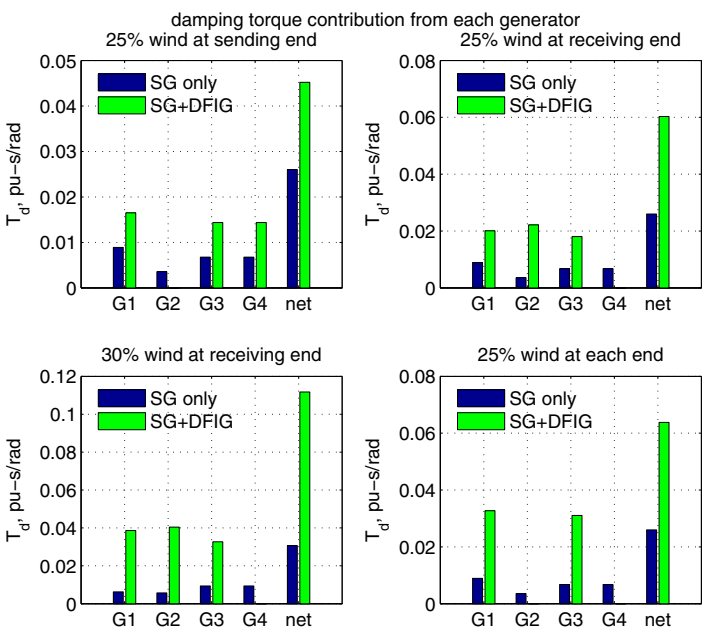

Fig. 6. Damping torque contribution (normalized with respect to speed) from individual generators across different wind penetration (in \% of system load) scenarios. System load (2800 MW) and tie-flow (400 MW) are kept constant

\section{B. SG vs DFIG: Higher system load, AC only}

In this section a typical planning scenario where future load growth would be supplied by additional wind is considered, whereby the conventional generation is kept constant. Output of wind farm G2 is increased from $700 \mathrm{MW}$ to $950 \mathrm{MW}$ to supply load increase from $2800 \mathrm{MW}$ to $3050 \mathrm{MW}$ distributed at buses 7 and 9 respectively, see Fig. 3 .

Fig. 7 compares the damping ratios with increased load supplied mostly by G2 which could be either SG or DFIG. Although the previous trend is visible, i.e. with DFIG the system gets better damped - the positive effect reduces at higher loading. After a threshold (around 3000 MW loading) the damping with DFIG is less than that with SG. Interestingly, the system frequency also becomes less with DFIG at such high loading despite reduction in generator inertia, see Fig. 8.
This can be attributed to increase in power angle due to reduction in voltage profile under such heavy loading scenario which dominates the reduction in inertia.

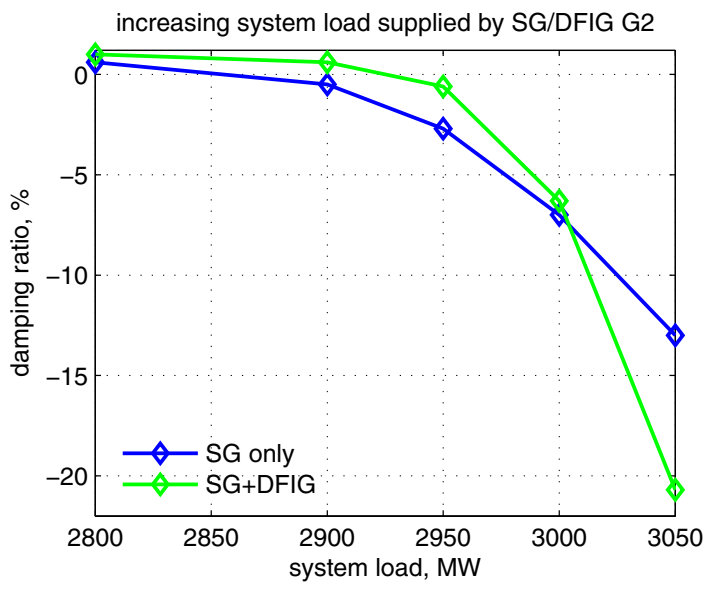

Fig. 7. Damping ratios of inter-area mode under different scenarios with increase in system load being supplied by increasing output of G2. Outputs of SGs, G1 and G4 are kept constant at their nominal values. Blue Trace: G2 is SG. Green Trace: G2 is DFIG

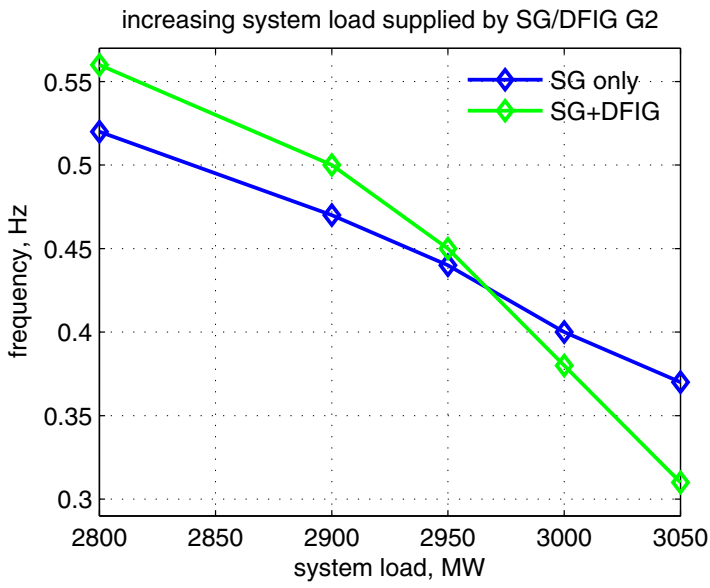

Fig. 8. Frequency of inter-area mode under different scenarios with increase in system load being supplied by increasing output of G2. Outputs of SGs, G1 and G4 are kept constant at their nominal values. Blue Trace: G2 is SG. Green Trace: G2 is DFIG

To understand the trend of damping ratios, damping torque analysis was repeated as in Section IV-A. The compass plot of torque variation of SGs normalized w.r.t the corresponding speed changes are shown in Fig. 9 for two loading conditions. Note that the the speed deviation $\Delta \omega$ of all generators will not be in same phase, but the objective being damping torque analysis - we are interested only in the phase difference of individual generator torque with respect to its own speed. Therefore the relative phase amongst the generator speeds is not illustrated in this figure.

At about $2950 \mathrm{MW}$ loading the magnitude of $\Delta T_{e}$ for $\mathrm{SG}$ (blue arrows) and DFIG (green arrows) are almost equal, see Fig. 9. However, the component of $\Delta T_{e}$ along $\Delta \omega$ is more negative for $\mathrm{SG}$ thereby producing poorer damping ratio. This is consistent with the individual damping torque contribution 
of generators shown in the top right subplot of Fig. 10 .

As the loading increases to about $3050 \mathrm{MW}$, the damping torque component in presence of DFIG becomes much more negative as compared to that with SG, see Figs 9 and 10. From Figs 7 and 10 the general trends of change in system damping can be explained form the magnitude of net damping torque under each condition.

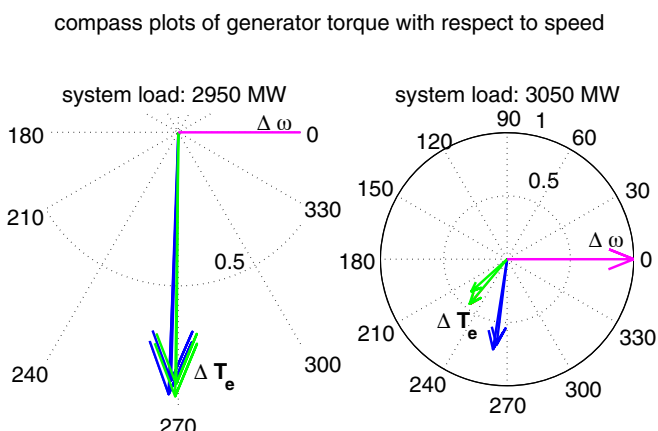

Fig. 9. Compass plots of torque deviations normalized with respect to speed change. Blue arrows: individual machine torques when all are SGs. Green arrows: individual machine torques when G2 is DFIG
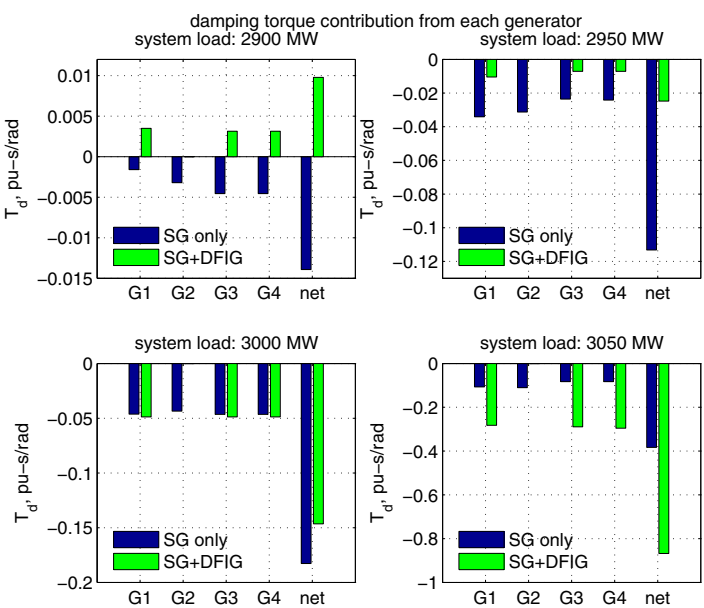

Fig. 10. Damping torque contribution (normalized with respect to speed) from individual generators with increase in system load being supplied by increasing output of G2. Outputs of SGs G1 and G4 are kept constant at their nominal values. Blue: G2 is SG. Green Trace: G2 is DFIG

\section{DFIG: Effect of HVDC Upgrade}

Under higher loading conditions the tie-flow increases from $400 \mathrm{MW}$ to about $550 \mathrm{MW}$. The effect of upgrading one of the existing AC tie-lines with a point-to-point VSC HVDC link was considered, see Fig. 3 (b). The objective of the dc link was to maintain flow through a single $\mathrm{AC}$ tie-line at its nominal value of $200 \mathrm{MW}$ and maintain unity power factor at either of the terminals with increasing wind penetration at G2.

Fig. 11 shows the system damping ratio with HVDC upgrade across different loading conditions. Under nominal loading with $400 \mathrm{MW}$ tie-flow and $2800 \mathrm{MW}$ load, inclusion of HVDC makes the system unstable. Such a trend of negative damping contribution continues till the tie-flow reaches 500 MW (2950 MW system load). However under heavier loading where DFIG starts producing negative impact on system damping, the HVDC upgrade is shown to produce considerable improvement. For example with $3000 \mathrm{MW}$ load the damping is improved from $-6.3 \%$ to about $-2 \%$ and under $550 \mathrm{MW}$ tie-flow (3050 MW load) from $-20.7 \%$ to $-2.5 \%$. On the other hand the system frequency is lesser with introduction of $\mathrm{dc}$ link up to the threshold of 2950 MW loading and increases with higher system load, see Fig. 12.

The reduction in damping with HVDC upgrade at normal loading (2800 MW) is primarily due to the fact that half the AC tie-flow (200 MW) behaves as constant power loads at buses 7 and 9 which contributes negatively [13]. With increased loading the dc link shares more power, thereby relieving the $\mathrm{AC}$ tie - which has a positive impact on system damping. When the tie-flow becomes more than $500 \mathrm{MW}$, this has a dominant contribution in increasing the damping ratio.

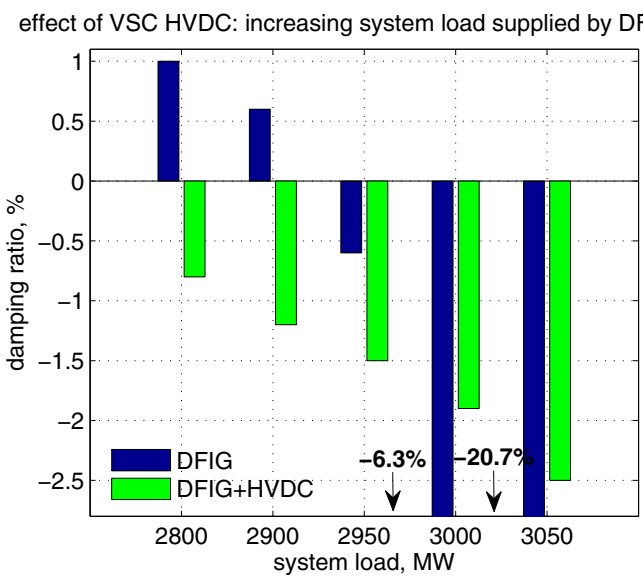

Fig. 11. DFIG with HVDC upgrade: Variation in damping ratio of interarea mode with increase in system load being supplied by increasing output of DFIG G2. Outputs of SGs G1 and G4 are kept constant at their nominal values

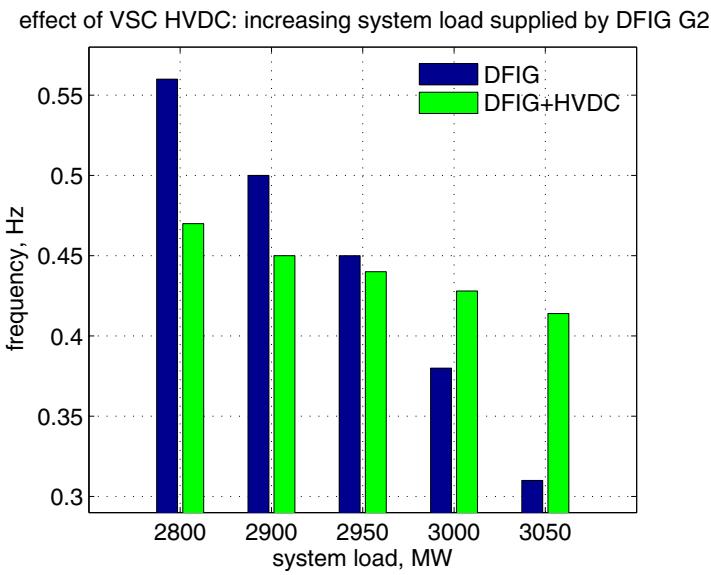

Fig. 12. DFIG with HVDC upgrade: Variation in frequency of inter-area mode with increase in system load being supplied by increasing output of DFIG G2. Outputs of SGs G1 and G4 are kept constant at their nominal values

In the above sections the system impact of DFIG is ana- 
lyzed considering typical operational and planning scenarios along with the effect of future HVDC upgrades. Most of the conclusions are in line with the results reported in literature for other systems with a few exceptions. Under heavy loading scenarios high wind penetration from the sending end of the tie is shown to have negative impact on system stability where HVDC upgrade shows beneficial effect. Detailed analysis of damping torque contribution from each generator is shown to provide better understanding of the trends. Power oscillation damping control through DFIG and/or VSC HVDC link is discussed in the next section.

\section{Power Oscillation Damping (POD) Controller}

Under 500 MW tie-flow condition (see Fig. 11), the system damping improves with inclusion of HVDC link but it still remains unstable. In this section power oscillation damping (POD) controller is considered to stabilize and improve system damping under this operating scenario.

\section{A. Control Design}

Unlike SISO decentralized control schemes proposed in literature, a SIMO centralized state-feedback controller based on pole-placement technique [14] was used in this paper. The voltage angle difference between buses 5 and 11 was employed as the remote feedback signal because of high observability. The POD for DFIG was used to modulate the $d$ and $q$ axes rotor currents while the POD for VSC HVDC modulated $P_{r e c}^{*}$ and $Q_{r e c}^{*}$, see Fig. 2 (c).

In state feedback framework the order of the controller becomes equal to that of the plant which may not be realistic for implementation. Therefore, balanced truncation [15] was used to simplify the system to a third order equivalent. As shown in Fig. 13 comparison of sigma plots [15] of full order vs reduced order system indicates that the inter-area mode is retained in the third order system which in turn was used for POD design. A settling time of $10 \mathrm{~s}$ was ensured through
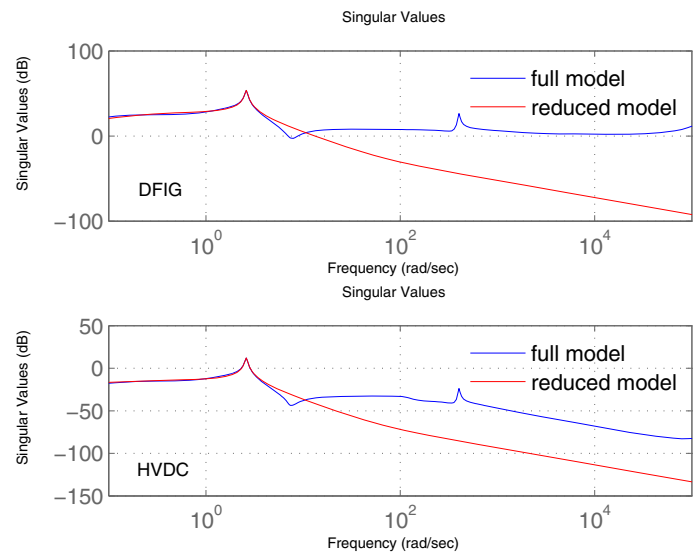

Fig. 13. Sigma plot of the full vs reduced order SIMO systems for DFIG and HVDC POD design

pole placement for either of DFIG and VSC HVDC PODs. An observer, five times faster than the desired oscillatory closed loop poles, was used to estimate the states. The parameters of the controllers in state space form are given the Appendix.

\section{B. Results with POD on DFIG}

Fig. 14 shows the dynamic performance of the system following a three-phase self-clearing fault near bus 8 for 80 ms under $550 \mathrm{MW}$ tie-flow condition. Without any POD the system is unstable for either of the scenarios where all generators are SGs (green trace: light grey in grey scale) and when G2 is DFIG (red trace: grey in grey scale). Supplementary POD control modulates $i_{d r}^{\prime *}$ and $i_{q r}^{\prime *}$ to ensure stabilization of the system and settling of the order of $10 \mathrm{~s}$. Presence of high frequency mode is visible in the rotor currents which is consistent with the sigma plot in Fig. 13. Modulation of rotor current leads to observable oscillations in $\omega_{r}$ and $\theta_{t w}$.
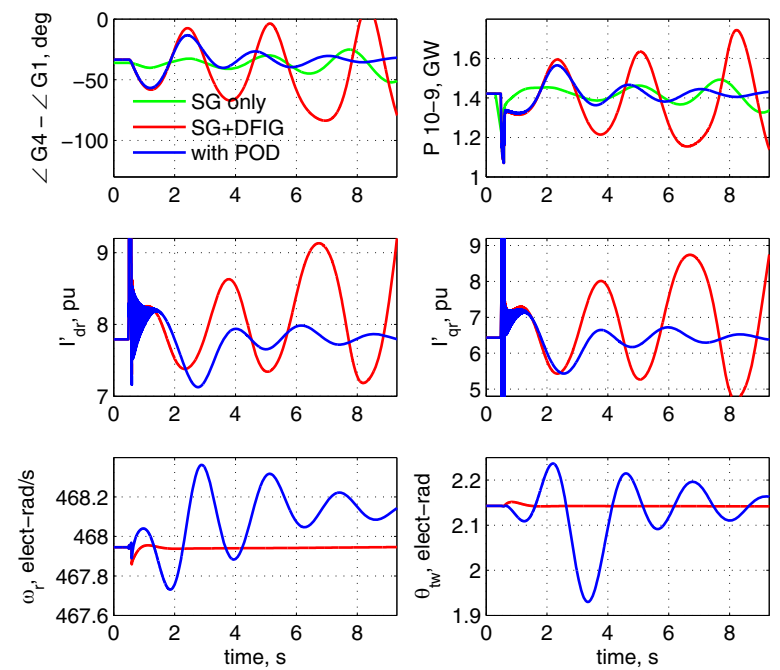

Fig. 14. Dynamic performance following a three-phase self-clearing fault near bus 8 for $80 \mathrm{~ms}$. Supplementary modulation of DFIG rotor currents is illustrated for power oscillation damping (POD)

\section{Results with POD on VSC HVDC}

Following a similar disturbance the system becomes unstable when no POD is in place while rectifier real power remains constant at about $350 \mathrm{MW}$ with unity power factor, see Fig. 15. When $P_{r e c}$ and $Q_{r e c}$ are modulated with HVDC POD the system gets well damped. Note that $V_{d c r e c}$ gets modulated in phase with $P_{r e c}$ while $V_{\text {dcinv }}$ remains constant due to inverterend dc link voltage control.

\section{CONCLUSION}

DFIGs were mostly found to contribute positively to system damping although the opposite trend was observed under certain scenarios. Various effects contributing to the overall system damping (e.g. wind penetration at sending vs receiving end etc.) were studied in isolation keeping the other factors (e.g. tie-flow etc.) constant. The findings were by and large similar to those reported in literature for other test systems with a few exceptions due to specific nature of case studies. Implications of supplying bulk of the future load growth by wind energy with only AC and both AC and HVDC were analyzed. Under heavy loading, high wind penetration at the sending end of the tie-line had negative impact on system 

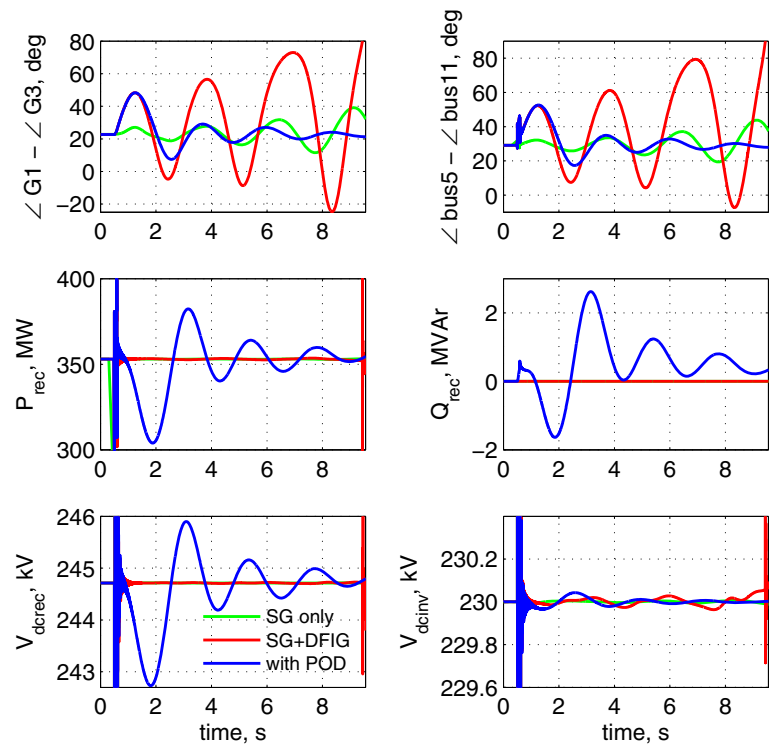

Fig. 15. Dynamic performance following a three-phase self-clearing fault near bus 8 for $80 \mathrm{~ms}$. Supplementary modulation of active and reactive power order of VSC HVDC is illustrated for power oscillation damping (POD)

stability while HVDC upgrade showed some beneficial effect. The trends were explained in terms of the damping torque contribution of synchronous generators which is the primary source of damping in power systems. Power oscillation damping (POD) control through supplementary modulation of DFIG rotor currents (both direct and quadrature axes) or active and reactive power order of VSC HVDC link was demonstrated.

There are several other factors that could possibly influence the overall dynamic performance which has been left out of this study. This includes different types and mix of excitation system, other modes (e.g. constant power factor) of wind farm control etc. Instead of complicating matters with too many factors changing at a time, the aim here was to rather isolate the effect of each. Nevertheless the above can be included in the same framework keeping the other factors constant to evaluate their individual effects, some of which has already been reported in the literature.

\section{APPENDIX}

TABLE I

PARAMETERS USED FOR DFIG MODELING

\begin{tabular}{|c|c||c|c|}
\hline Parameters & Values $(\mathrm{pu})$ & Parameters & Values \\
\hline \hline$R_{s}$ & 0.00488 & $\omega_{s}$ & $1.0 \mathrm{pu}$ \\
\hline$L_{s s}$ & 4.0452 & $c_{s h}$ & 0.09 pu-s/elect. rad \\
\hline$L_{m}$ & 3.95279 & $k_{s h}$ & $0.3 \mathrm{pu} /$ elect. rad \\
\hline$L_{r r}$ & 4.0523 & $H_{g}$ & $3.5 \mathrm{~s}$ \\
\hline$R_{r}$ & 0.00549 & $\omega_{\text {rrated }}$ & $1.3 \mathrm{pu}$ \\
\hline
\end{tabular}

TABLE II

DFIG PRIMARY CONTROLLER PARAMETERS

\begin{tabular}{|c|c|}
\hline Parameters & Values \\
\hline \hline$K(s)$ & $-300\left(\frac{R_{r}+s \sigma L_{r r}}{s}\right)$ \\
\hline$K_{v c}$ & 20.0 \\
\hline
\end{tabular}

TABLE III

HVDC PARAMETERS

\begin{tabular}{|c|c||c|c|}
\hline Parameters & Values & Parameters & Values \\
\hline \hline$V_{d \operatorname{cinv}}$ & $230 \mathrm{kV}$ & $C$ & $0.1 \mathrm{mF}$ \\
\hline$L_{c}$ & $10.26 \mathrm{mH}$ & $L_{L}$ & $680 \mathrm{mH}$ \\
\hline$R_{c}$ & $0.0 \mathrm{ohm}$ & $R_{L}$ & $10.2 \mathrm{ohm}$ \\
\hline
\end{tabular}

$$
\begin{gathered}
K_{D F I G}=\left[\begin{array}{rrr|r}
-0.6403 & -625.8327 & -60.0927 & -0.1050 \\
-0.0018 & -4.3285 & -1.8699 & -0.0007 \\
0.0052 & 7.4486 & -0.6272 & 0.0004 \\
\hline 1.0105 & 100.1310 & -181.4882 & 0 \\
1.4912 & 136.2052 & -246.8745 & 0
\end{array}\right] \\
K_{H V D C}=\left[\begin{array}{rrrr}
-0.0005 & 33.1850 & 3.1831 & 0.1782 \\
-0.0062 & -4.3669 & -1.8311 & -0.0235 \\
0.0097 & 6.8996 & -0.6497 & 0.0095 \\
\hline 1.5765 & 205.1994 & -304.6941 & 0 \\
-0.7543 & 11.3545 & -16.8749 & 0
\end{array}\right]
\end{gathered}
$$

\section{REFERENCES}

[1] J. J. Sanchez-Gasca, N. W. Miller, and W. W. Price, "A modal analysis of a two-area system with significant wind power penetration," in IEEE PES Power Systems Conference and Exposition, 2004., 2004, pp. 11481152 vol.2.

[2] G. Tsourakis, B. M. Nomikos, and C. D. Vournas, "Contribution of doubly fed wind generators to oscillation damping," IEEE Transactions on Energy Conversion, vol. 24, no. 3, pp. 783-791, 2009.

[3] E. Hagstrm, I. Norheim, and K. Uhlen, "Large-scale wind power integration in norway and impact on damping in the nordic grid," Wind Energy, vol. 8, no. 3, pp. 375-384, 2005.

[4] D. J. Vowles, C. Samarasinghe, M. J. Gibbard, and G. Ancell, "Effect of wind generation on small-signal stability; a new zealand example," in proceedings of IEEE Power and Energy Society General Meeting, 2008, pp. 1-8.

[5] F. M. Hughes, O. Anaya-Lara, N. Jenkins, and G. Strbac, "Control of dfig-based wind generation for power network support," IEEE Transactions on Power Systems, vol. 20, no. 4, pp. 1958-1966, 2005.

[6] H. F. Latorre, M. Ghandhari, and L. Soder, "Use of local and remote information in POD control of a VSC-HVDC," in proceedings of PowerTech, 2009 IEEE Bucharest, 2009, pp. 1-6.

[7] R. Si-Ye, L. Guo-Jie, and S. Yuan-Zhang, "Damping of power swing by the control of VSC-HVDC," in proceedings of IPEC 2007 International Power Engineering Conference, 2007, pp. 614-618.

[8] N. R. Chaudhuri and B. Chaudhuri, "Damping and relative modeshape estimation in near real-time through phasor approach," IEEE Transactions on Power Systems, vol. 26, no. 1, pp. 364-373, 2011.

[9] P. Kundur, Power system stability and control, ser. The EPRI power system engineering series. New York; London: McGraw-Hill, 1994.

[10] J. G. Slootweg, H. Polinder, and W. L. Kling, "Dynamic modelling of a wind turbine with doubly fed induction generator," in IEEE Power Engineering Society Summer Meeting, 2001, vol. 1, 2001, pp. 644-649 vol.1.

[11] R. Pena, J. C. Clare, and G. M. Asher, "Doubly fed induction generator using back-to-back pwm converters and its application to variablespeed wind-energy generation," IEE Proceedings on Electric Power Applications, vol. 143, no. 3, pp. 231-241, 1996.

[12] C. Schauder and H. Mehta, "Vector analysis and control of advanced static var compensators," IEE Proceedings on Generation, Transmission and Distribution, vol. 140, no. 4, pp. 299-306, 1993.

[13] M. Klein, G. Rogers, and P. Kundur, "A fundamental study of inter-area oscillations in power systems," IEEE Transactions on Power Systems, vol. 6, no. 3, pp. 914-921, 1991.

[14] A. Zolotas, B. Chaudhuri, I. Jaimoukha, and P. Korba, "A study on LQG/LTR control for damping inter-area oscillations in power systems," IEEE Transactions on Control Systems Technology, vol. 15, no. 1, pp. 151-160, 2007.

[15] S. Skogestad and I. Postlethwaite, Multivariable feedback control: analysis and design. Chichester: Wiley, 1996. 